\title{
II Signor Cestoni alla prova delle pignatte
}

\author{
Francesco Luzzini
}

Dipartimento di Studi Umanistici,Vercelli - Università degli Studi del Piemonte Orientale francesco_luzzini@yahoo.com; francesco.luzzini@lett.unipmn.it

Leggendo gli epistolari scientifici dei decenni a cavallo tra XVII e XVIII secolo non è raro imbattersi in personaggi " $\mathrm{mi}$ nori" come speziali, medici di provincia, chirurghi, membri del basso clero, che si dedicarono con passione ai più disparati campi delle scienze mediche e naturali. Individui magari estranei alle accademie, alle università ed ai più o meno raffinati salotti mondani; ma che spesso dimostrarono di possedere competenze tecniche e scientifiche notevolissime e paragonabili, se non superiori, a quelle vantate da molti dei loro più blasonati colleghi. Troppo a lungo la storiografia tradizionale ha sottovalutato o ignorato il valore di questi tecnici, che dei dibattiti scientifici della loro epoca furono protagonisti a tutti gli effetti. Non è un caso che la loro rivalutazione, negli ultimi decenni, sia dipesa da una maggiore sensibilità degli storici per una scienza fatta d'esperienze, e non solo di teorie. È stato così possibile comprendere l'enorme importanza di questi soggetti, che per la lunga e immeritata trasparenza all'attenzione degli studiosi sono stati appropriatamente definiti come «figure invisibili» (Generali, 2004).

Un caso emblematico è rappresentato da Diacinto Cestoni (1637-1718), marchigiano di nascita, ma che visse ed operò a Livorno fino alla sua morte (Fig. 1). E invisibile, Cestoni, lo fu davvero a lungo, prima che recenti lavori ne rivendicassero $i$ meriti. Ma in vita la sua fama fu tutt'altro che trascurabile: ne sono un'eloquente testimonianza le sue prestigiose corrispondenze, tra cui figurano scienziati del calibro di Marcello Malpighi (1628-1694), Francesco Redi (1626-1697) e Antonio Vallisneri (1661-1730).

È proprio negli epistolari che troviamo le prove decisive della grandezza scientifica di questo singolare personaggio, che pubblicò pochissimo, ma le cui ricerche esercitarono un'influenza tutt'altro che trascurabile su diversi dibatti mediconaturalistici del suo tempo (Generali, 2004; Generali, 2007; Generali, 2010; Luzzini, 2011; Tanga, 2007). Questo «uоmо grande» (Vallisneri, 1991), che era solo uno speziale - vale a dire un farmacista - ma che, a dire del Redi, ne sapeva «più

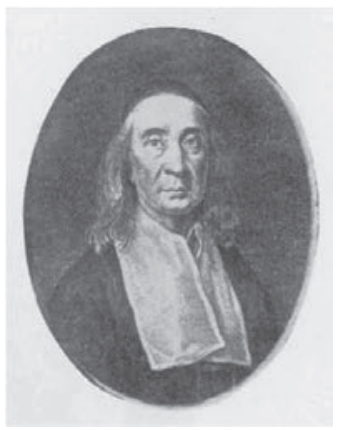

Fig. 1 - Diacinto Cestoni. di quaranta medici», si potrebbe descrivere a ragione come un paladino della ricerca sperimentale basata sulle sole esperienze, libera da qualsiasi velleità filosofica. Autodidatta, la mancanza d'una formazione accademica non gli impedì d'acquisire una competenza scientifica vastissima, che gli permise d'affermarsi come uno dei maggiori microscopisti italiani e di spaziare coi suoi studi dalla zoologia alla botanica, dalla medicina alle scienze della Terra. In quest'ultimo campo Cestoni riuscì a dare un contributo fondamentale a un tema d'indagine assai dibattuto a quell'epoca: l'origine delle fonti. E lo fece grazie alla sua corrispondenza con Vallisneri, che lo teneva in grandissima stima e che di questo dibattito fu l'indiscusso protagonista.

Si è già parlato su queste pagine (Luzzini, 2012) della diatriba che fra Sei e Settecento opponeva i sostenitori dell'origine esclusivamente meteorica delle acque sorgenti ai difensori dei lambicchi, secondo i quali era possibile che le acque marine potessero desalinizzarsi filtrando attraverso gli strati rocciosi. Cestoni, che era contrarissimo a questa seconda ipotesi, scrisse ripetutamente e senza mezzi termini a Vallisneri che «l'acqua salata di tutto l'universo» non si poteva «ridurla dolce, e bevibile per via di fultrazione (...). La mia professione è maestra infallibile di tal dottrina, e so di certo, che a voler ridurre dolce l'acqua salata, è necessario distillarla: e questo lavoro del distillare lo fa incessantemente il calor solare, et aereo in tutto l'universo, e poi per via di piogge torna a venir di nuovo a basso: sicché asseverantemente le piogge sono l'alimento delle fontane, e fiumi di questo mondo» (Cestoni, 1940).

Vallisneri, si sa, era dello stesso parere. Ma come dimostrare che le rocce e la terra non potevano sottrarre il sale all'acqua? La questione premeva molto al medico emiliano, che, se fosse riuscito a dare prove certe dell'impossibilità di questo processo, avrebbe agevolato di molto la sua teoria sull'origine delle fonti. E fu proprio Cestoni a dargli lo spunto per risolvere l'enigma, coinvolgendolo nell'esecuzione di un'efficacissima prova sperimentale. Scrivendo all'amico nel gennaio 1698, gli rivelò d'avere «accomodata una bombola (della quale qui accluso vedrà la figura) di terra bianca non invetriata, e l'bo serrata con cera, e poi l'ho legata ad un sasso, e l'ho messa in questo fosso dell'acqua salata, che circonda il lazzaretto, quale terrò così sin a lunedi prossimo (...), e vedrò se vi sarà penetrata acqua, ma io dubbito di no! Lei faccia pure l'esperienza della pentola in acqua acconcia col sale; perchè o più sale, o meno sale, sempre torna la medesima esperienza; poiché ancor l'acqua del mare varia la sua salsedine essendo talvolta più salata, et alle volte meno salata, procedendo essa variazione quando dalle piogge, quando dalle correnti, quando dalle fumane vicine (...). Dentro alla bombola, che ho messo nell'acqua 
salata non vi troveremo acqua di nessuna sorte, né dolce, né salza» (Cestoni, 1940) (Fig. 2).

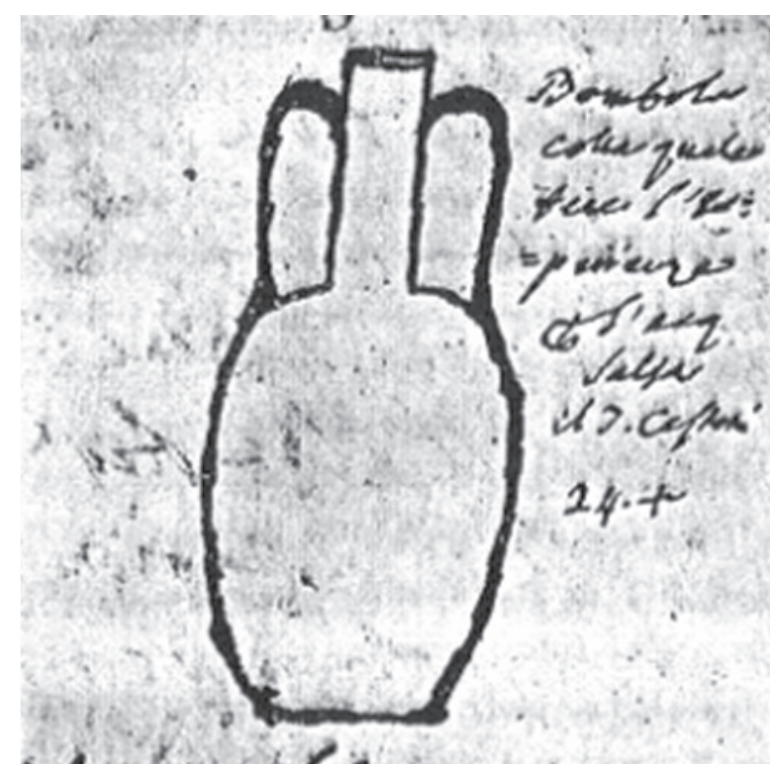

Fig. 2 - La bombola.

In verità di giorni ne passarono dieci, dal momento che il lunedì prestabilito ci fu un «tempo tanto strano» che allo speziale non fu possibile ripescare la bombola. L'impresa gli riuscì tre giorni dopo: aperto il contenitore, a dispetto del suo pronostico, vi trovò dentro «sei dramme d'acqua limpida cristallina». Ma questa era ancora salata, ed al sapore non era «diversificata punto» da quella marina (Cestoni, 1940).

Vallisneri, da parte sua, fece preparare «una palla vuota di terra da pignatte» e la immerse in un recipiente colmo d'acqua salata, dove «tardò otto giorni a trapellar l'acqua» («perché l'aveo fatta ben cuocere nella fornace»). Una volta ripescata e rotta la palla, vi trovò «l'acqua salsa penetrata senza perdere il sale». Subito scrisse «a Livorno al Sig.r Cestoni», mettendolo al corrente del risultato; e apprese con gioia che anche l'amico, nel suo «vaso di certa terra barbara poroso», aveva trovato acqua salata (Vallisneri, 1694-1712). Fu così che i due esperimenti, eseguiti indipendentemente l'uno dall'altro, confutarono la teoria dei lambicchi. Era ormai chiaro, come Cestoni aveva più volte sostenuto, che «l'acqua del mare dolcifica solo per destillazione, e non per filtrazione» (Vallisneri, 1694-1712): non dal mare provenivano le sorgenti, ma dalle piogge.

La collaborazione di Cestoni fu essenziale per Vallisneri, che nella sua Lezione Accademica intorno all'Origine delle Fontane (Vallisneri, 1715) utilizzò la prova delle pignatte come uno degli argomenti più validi per attaccare la teoria dei lambicchi. Ma i raffinati palati della Repubblica delle Lettere di primo Settecento non si sarebbero accontentati delle sole prove sperimentali. Occorreva che queste fossero inquadrate in un sistema teoricamente ben definito perché la comunità dei savants europei le potesse prendere in seria considerazione. Questo compito, ovviamente, spettava a Vallisneri, che ad un adamantino spe- rimentalismo univa competenze (e ambizioni) filosofiche non marginali. Cestoni, ben lieto d'ignorare le diatribe accademiche, saccontentò di tornare al «non men utile che dilettevole studio dell'istoria naturale»(Cestoni, 1940): dove, che piacesse o no ai filosofi, l'unico modo per verificare le cose supposte era di provarle con le sperienze.

\section{BIBLIOGRAFIA}

Cestoni D. (1940) - Epistolario ad Antonio Vallisneri. Parte I, con introduzione ed a cura di Silvestro Baglioni, Reale Accademia d'Italia, Roma.

Generali D. (2004) - Uno speziale che «superava la sua condizione». Il caso dell'invisibilità postuma di Diacinto Cestoni. In: Monti M.T., Ratcliff M. (eds), Figure dell'invisibilità. Le scienze della vita nell'Italia d'Antico Regime, Olschki, Firenze, pp. 83-118.

Generali D. (2007) - Antonio Vallisneri. Gli anni della formazione e le prime ricerche. Olschki, Firenze, pp. 319-350.

Generali D. (2010) - Diacinto Cestoni. Uno speziale sei-settecentesco tra invisibilità e riscoperta storiografica. Vita pensata, Rivista mensile di filosofia 2010, 3: 35-37.

Luzzini F. (2011) - Multa curiosa. Vallisneri's early studies on Earth sciences. Nuncius 2011, 2: 334-354.

Luzzini F. (2012) - Contro i lambicchi. Antonio Vallisneri e l'origine delle sorgenti. Acque Sotterranee 2012, 1: 83-84. DOI: 10.7343 / AS-011-12-0011

Tanga M. (2007) - Giacinto Cestoni, i rapporti con Redi e le scienze della vita nel XVII secolo. Tesi di dottorato, Università degli Studi di Pisa, Facoltà di Lettere e Filosofia, Dottorato di ricerca in Storia della Scienza.

Vallisneri A. (1694-1712) - Quaderni di osservazioni, voll. I-VII, Quaderno V. Manoscritto, Biblioteca Estense, Modena.

Vallisneri A. (1715) - Lezione Accademica intorno all'Origine delle Fontane. Appresso Gio. Gabbriello Ertz, Venezia.

Vallisneri A. (1991) - Epistolario. Volume I (1679-1710), a cura di Dario Generali, Franco Angeli, Milano, p. 178.

www.francescoredi.it/Database/redi/redi.nsf/b4604a8b566ce010 c125684d00471e00/57a2c8d4c4d34706c12569f40039fcf7! OpenDocument 